Brazilian Journal

of Chemical

ISSN 0104-6632

Engineering

\title{
MAGNETIC RESONANCE IMAGING (MRI): A TECHNIQUE TO STUDY FLOW AN MICROSTRUCTURE OF CONCENTRATED EMULSIONS
}

\author{
M. A. d'Avila ${ }^{1 *}$, R. L. Powell ${ }^{2}$, R. J. Phillips ${ }^{2}$, N. C. Shapley ${ }^{3}$, \\ J. H. Walton ${ }^{4}$ and S. R. Dungan \\ ${ }^{1}$ Laboratório de Modelagem e Simulação de Processos Químicos, Departamento de Processos \\ Químicos, Faculdade de Engenharia Química, Universidade Estadual de Campinas \\ (UNICAMP), Av. Albert Einstein 500, 13083-970, Campinas - SP, Brasil. \\ E-mail: madavila@feq.unicamp.br \\ ${ }^{2}$ Department of Chemical Engineering and Materials Science \\ University of California, 95616, Davis - CA, USA. \\ ${ }^{3}$ Department of Chemical Engineering, Columbia University, NY \\ ${ }^{4}$ NMR Facility, University of California, Davis - CA, USA \\ ${ }^{5}$ Department of Chemical Engineering and Materials Science and Department \\ of Food Science and Technology, University of California, Davis - CA, USA.
}

(Received: January 20, 2004 ; Accepted: July 28, 2004)

\begin{abstract}
Nuclear magnetic resonance (NMR) and magnetic resonance imaging (MRI) have recently been recognized as important techniques for $R \& D$ of products and processes, as is attested by several successful applications in different areas of chemical engineering in recent years. In this article we present new experimental methods based on MRI to study flow and microstructure of concentrated emulsions. The objective is to present the unique features of this noninvasive technique to accurately measure different properties of flowing particulate opaque systems. Experimental results of velocity profiles, spatial distribution of droplet sizes and spatial homogeneity of an oil-in-water dispersion in a horizontal, concentric cylinder geometry using different pulse sequences are presented. The application of these techniques allowed probing important information on flow and microstructure of multiphase systems of interest in chemical engineering and food science.
\end{abstract}

Keywords: Concentrated emulsions; Magnetic resonance imaging; Velocity profiles; Concentration distribution; Droplet size distribution.

\section{INTRODUCTION}

Nuclear magnetic resonance (NMR) and magnetic resonance imaging (MRI) are techniques traditionally used by spectroscopists to study structure and conformation of molecules and by medical doctors as a tool for clinical diagnosis, respectively. In recent years, these techniques have been recognized to have great potential as an experimental tool for engineering science research due to their versatility and accuracy, as is attested by the considerable interest shown in their use as an $\mathrm{R} \& \mathrm{D}$ tool in product and process development (Gladden, 2003; Mantle and Sederman, 2003). Applications identified in different fields of chemical engineering range from chemical reaction to food and biochemical engineering (Gladden, 2003). Although these techniques have been used mostly in

*To whom correspondence should be addressed 
laboratory research due to their high operational costs, low field NMR and MRI equipments have shown to be able to perform routine process and quality control in industry (Gladden, 2003; Goudappel et al., 2001; Arola et al., 1997) and the potential for use of these techniques to solve industrial problems is widely recognized (Hall et al., 2001).

Nuclear magnetic resonance (NMR) consists in the application of radio-frequency (r.f.) pulses in conjunction with a static magnetic field to obtain spectral information on the molecular species in the sample. On the other hand, MRI consists of an NMR equipped with magnetic field gradients to obtain simultaneously spatial resolution and dynamic information such as diffusion and flow inside the sample (Callaghan, 1991). Important features of MRI-based techniques are that they are noninvasive, are suited for opaque systems and allow spatially resolved measurement. These features pose MRI as an important tool for the study of flow and microstructure of concentrated emulsions which, due to their inherent opacity, cannot be studied in-situ using the usual methods that require sample transparency, e.g., optical microscopy, static light scattering, video imaging and laser-Doppler velocimetry.

Emulsions can be defined as a physical system composed of two liquid phases where one phase is dispersed into the other in the form of droplets (Binks, 1998). These systems are of great scientific and technological interest and applications are widely found in the pharmaceutical, food, oil and polymer processing industries. Recent interest in flow of such systems involves a wide variety of topics ranging from food (Walkenstrom and Hermansson, 2002) and polymer processing (Velankar et al., 2001) to shear banding in viscometric flows (Salmon et al., 2003) and blood substitutes for drug delivery (Riess, 2002). Many industrial processes involving emulsions in the food, polymer and oil industries deal with flow of concentrated emulsions, which have characteristics that differ considerably from those of single-phase flows. It is well known that the flow properties of concentrated emulsions are strongly controlled by the microstructure of drops and interparticle interactions created by hydrodynamic and colloidal forces within the fluid (Loewenberg, 1998; Zinchenko and Davis, 2002). External forces, such as shear and gravity, as well as the geometry can strongly affect the overall flow of emulsions and suspensions (Shapley et al., 2003; d'Avila, 2003).

Up to the present, applications to study flow of emulsions are limited and the capabilities of different
MRI methods to study these systems have not been fully explored (Newling et al., 1997; d'Avila, 2003). On the other hand, recent results demonstrate the flexibility of this technique to characterize flow and rheology of dispersed systems (Shapley et al., 2003; Chow et al., 1994; Abbott et al., 1991; Seymour et al., 1993), foods (Choi et al., 2002), polymer melts (Uludag et al., 2001), stratified fluids (Jeong et al., 1994), Taylor instability (Seymour et al., 1999), viscometric flows of complex fluids (Britton et al., 1999), turbulent flows (Li et al., 1994), flow in porous media (Mair et al., 2001; Packer, 2003), and mixing (McCarthy et al., 2002; d'Avila et al., 2003). The study of emulsions under static conditions with NMR has also been explored to gather important information such as droplet size distribution (Ambrosone et al., 1999) and destabilization mechanisms such as creaming (Newling et al., 1997), coalescence (Lee et al., 1998) and Ostwald ripening (Hedin and Furo, 2001).

The purpose of this article is to describe the methodology used to determine flow, spatial homogeneity and microstructure of concentrated oilin-water emulsions through MRI methods, showing the capabilities of this technique to perform these measurements accurately. Oil-in-water emulsions are widely used as model systems for foods and biological systems, and research in rheology and fluid mechanics has been conducted in order to understand the effects of the microstructure in the flow behavior and vice-versa (Mishra, 1998; Otsubo and Prud'homme, 1994). Horizontal concentric cylinders were used to generate viscometric flows and are suitable for studying the effects of flow on the fluid structure of two-phase, dispersed fluids (Phillips et al., 1992; Tirumkudulu et al., 1999). Imaging methods based on this technique were successfully implemented and allowed measurement of velocity profiles, concentration maps and droplet size distributions with spatial resolution.

In addition to the specific applications presented in this article, we believe that use of these novel MRI-based methodologies will permit advance significantly the understanding of systems involving flowing emulsions and other dispersed systems not contemplated here.

\section{MRI METHODS}

\section{Velocity Measurements}

The two main methods for measuring velocities using MRI are time-of-flight (TOF) and phase- 
encoding imaging. In this article, we have focused on the TOF method (Axel et al., 1986) and details on the phase-encoding method can be found elsewhere (Pope and Yao, 1993; Fukushima, 1999; Shapley et al., 2003). Time-of-flight velocimetry (TOF) consists of saturating a predetermined region of a flowing system by applying a radio-frequency (r.f.) pulse to null the signal of this region. A slice is taken in the direction perpendicular to the main flow. The displacement of the tagged nuclei is obtained after a known period of time, and the velocity is determined from the ratio of displacement to time. The advantage of this technique is that it is easy to implement and it gives accurate velocity measurements at steady state. However, it cannot

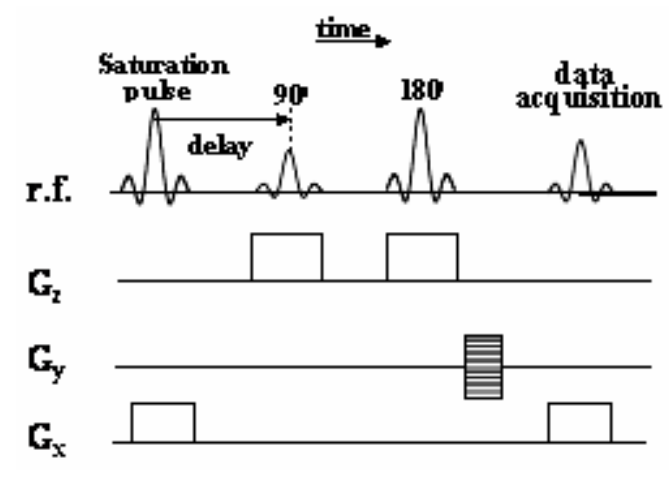

Figure 1: TOF pulse sequence. The first r.f. pulse corresponds to the saturation pulse that nulls the tag signal. The subscripts $\mathrm{x}, \mathrm{y}$ and $\mathrm{z}$ correspond to the coordinate directions shown in Figure 1.

\section{Droplet Size Distribution Measurements}

When droplets sizes are on the millimeter scale, it is not difficult to obtain an image of the droplets using conventional MRI methods. However, emulsions have particle sizes that range from 0.1 to $50 \mu \mathrm{m}$. Therefore, methods based on the selfdiffusion properties of the molecules confined in the droplets such as pulsed-field gradients should be used. Here, droplet size distribution was measured using pulsed-field gradient stimulated-echo pulse sequences (PGSTE) (Callaghan, 1991). This pulse sequence has the advantage of avoiding signal loss due to spin-spin relaxation $\left(\mathrm{T}_{2}\right)$ and it consists in the resolve flows at low velocities $(<0.1 \mathrm{~mm} / \mathrm{s})$ and it is limited to unidirectional flow. Other methods based on tagging such as the DANTE pulse sequence (Callaghan, 1991) have been used in order to obtain steady-state flow fields in two dimensions (Jeong et al., 1994). Figure 1 shows a TOF pulse sequence. In this figure, the first r.f. pulse corresponds to the saturation pulse, which nulls the signal of the region specified by the gradient, in this case the $\mathrm{x}$ direction gradient $G_{x}$. The remaining pulse sequence corresponds to the usual spin-echo imaging pulse sequence. The magnetic field gradients $G_{x}, G_{y}$ and $\mathrm{G}_{\mathrm{z}}$ are the frequency-encoding, phase-encoding and slice-selection gradients, respectively, and they have units of magnetic field divided by length.

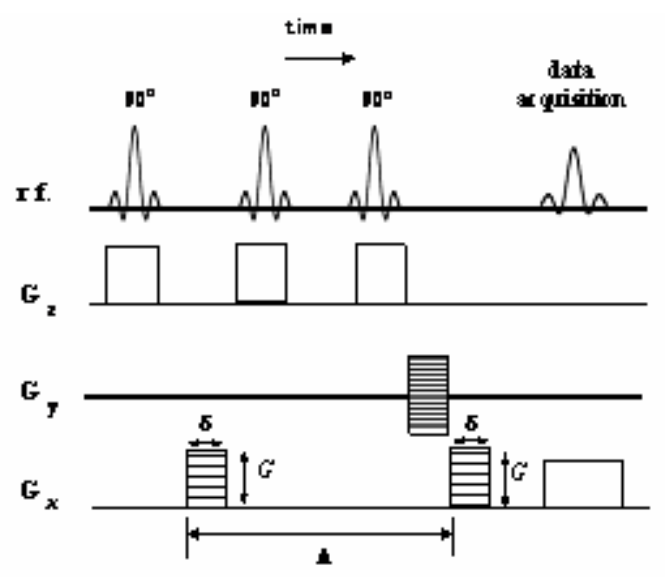

Figure 2: PGSTE pulse sequence. $G$ is the gradient intensity, $\delta$ is the gradient duration, $\Delta$ is the diffusion time.

application of three $90^{\circ}$ r.f. pulses and two pulsed gradients with magnitude $\mathrm{G}$ and duration $\delta$ separated by an interval of time $\Delta$ (Figure 2$)$. The relative signal decay function $\mathrm{S} / \mathrm{S}_{0}$, assuming a Gaussian phase distribution of spins for random motion of molecules confined in a sphere of radius a, is given by the following expression (Packer and Rees, 1972):

$$
\begin{aligned}
& \mathrm{S} / \mathrm{S}_{0}=\mathrm{R}(\mathrm{g}, \delta, \Delta, \mathrm{D}, \mathrm{a})= \\
& =\exp \left[-2{\gamma_{\mathrm{G}}}^{2} \mathrm{G}^{2} \sum_{\mathrm{m}=1}^{\infty} \mathrm{f}\left(\alpha_{\mathrm{m}}\right)\right]
\end{aligned}
$$


and

$$
\begin{gathered}
\mathrm{f}\left(\alpha_{\mathrm{m}}\right)=\left[\alpha_{\mathrm{m}}^{2}\left(\alpha_{\mathrm{m}}^{2} \mathrm{a}^{2}-2\right)\right]^{-1}\left[2 \delta / \alpha_{\mathrm{m}}^{2} \mathrm{D}-\left[2+\exp \left(-\alpha_{\mathrm{m}}^{2} \mathrm{D}(\Delta-\delta)-2 \exp \left(-\alpha_{\mathrm{m}}^{2} \mathrm{D} \delta\right)\right] /\left(\alpha_{\mathrm{m}}^{2} \mathrm{D}\right)^{2}-\right.\right. \\
\left.-\left[2 \exp \left(-\alpha_{\mathrm{m}}^{2} \mathrm{D} \Delta\right)++\exp \left(-\alpha_{\mathrm{m}}^{2} \mathrm{D}(\Delta+\delta)\right)\right] /\left(\alpha_{\mathrm{m}}^{2} \mathrm{D}\right)^{2}\right]
\end{gathered}
$$

Here, $S_{0}$ is the signal when $G=0$, D is the selfdiffusion coefficient, $\gamma_{\mathrm{G}}$ is the gyromagnetic ratio and $\alpha_{\mathrm{m}}$ are roots of the Bessel function equation $\alpha_{\mathrm{m}} \mathrm{aJ}_{3 / 2}^{\prime}\left(\alpha_{\mathrm{m}} \mathrm{a}\right)-(1 / 2) \mathrm{J}_{3 / 2}\left(\alpha_{\mathrm{m}} \mathrm{a}\right)=0$.

If the system is polydisperse, the signal decay is due to contributions from droplets of different sizes, and then the signal attenuation is given by the volume average over all sizes as

$$
\begin{aligned}
& \left(\mathrm{S} / \mathrm{S}_{0}\right)_{\text {obs }}= \\
& =\int_{0}^{\infty} \mathrm{a}^{3} \mathrm{P}(\mathrm{a}) \mathrm{R}(\mathrm{D}, \mathrm{g}, \delta, \Delta, \mathrm{a}) \mathrm{da} / \int_{0}^{\infty} \mathrm{a}^{3} \mathrm{P}(\mathrm{a}) \mathrm{da}
\end{aligned}
$$

Here $P(a)=1 /\left(8 a^{2} \sigma^{2} \pi\right)^{1 / 2} \exp \left[-(\ln 2 a-\ln 2 \bar{a})^{2} /\left(2 \sigma^{2}\right)\right]$ is a lognormal size distribution function with mean radius $\overline{\mathrm{a}}$ and standard deviation $\sigma$. Fitting equations (1) to (3) to experimental values of $\left(S / S_{0}\right)$ gives $\bar{a}$ and $\sigma$ for the particle size lognormal distribution.

The limitation of this method is that it is necessary to assume an a priori size distribution, in this case, a lognormal distribution. This does not present a problem for the case of emulsions, where this distribution is usually seen. Data processing efforts based on regularization methods in order to determine size distributions without assuming a known distribution have appeared in the literature (Hollingsworth and Johns, 2003), and it is a promising area for the improvement of pulsed-field gradient methods.

\section{Volume Fraction Measurements}

Volume fraction distributions of droplets can be obtained using chemical shift imaging (CSI). The chemical shift expresses the fine structure of resonance lines and depends on the electronic environment of the nuclei. Therefore, it is possible to associate a determined molecule or molecular group by its chemical shift. Spectroscopy techniques used to identify and assign chemical structures are largely based on chemical shifts (Slichter, 1990). In the case of this work, the continuous and dispersed phases could be identified by two distinct peaks. Peak intensity in the spectrum is proportional to the number of spins in the sample; i.e., it is proportional to the concentration. If magnetic field gradients are applied, NMR spectra can be obtained for each position in the sample. With this information, a quantitative map of volume fractions can be obtained. The application of imaging gradients to obtain an NMR spectrum at each pixel is known as chemical shift imaging (CSI) (Rumpel and Pope, 1993). Figure 3 shows the CSI pulse sequence used in this work, which consists of the application of a spin-echo pulse sequence together with phaseencoding gradients $G_{x}$ and $G_{y}$, and a slice-selection gradient $\mathrm{G}_{\mathrm{z}}$. Other methods based on signal intensity can be used to determine concentration distributions in dispersed systems (McCarthy et al., 2002; Corbett et al., 1995; Bobroff and Phillips, 1998), but in the case of emulsions, where both phases contribute to the total signal, these methods have to rely on signal suppression such as selective excitation.

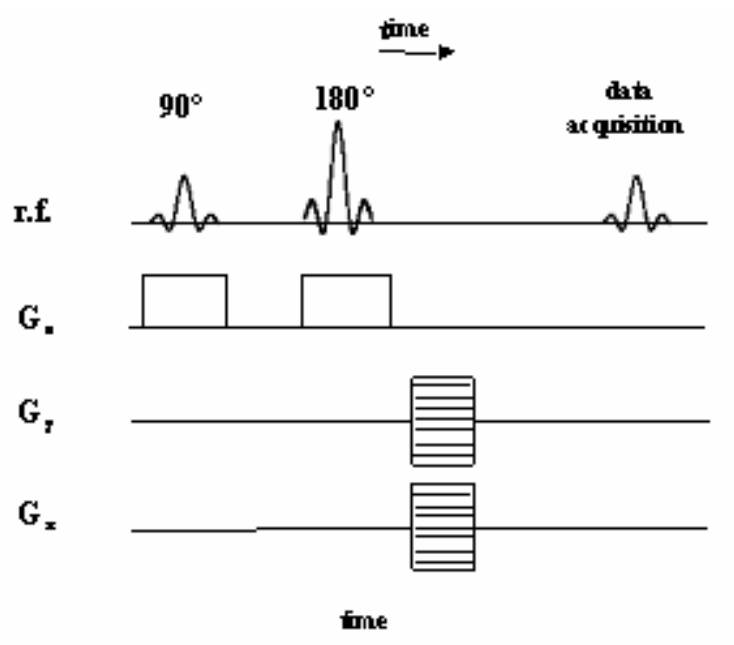

Figure 3: Chemical shift imaging pulse sequence. 


\section{EXPERIMENTAL}

The emulsions used in this study were composed of isooctane-in-water, with oil volume fractions $\phi_{0}$ of 0.3 and 0.4. Here, oil volume fraction is defined as the volume of the isooctane phase divided by the total volume. Isooctane was purchased from Fisher Scientific (Fair Lawn, NJ). The emulsions were stabilized with 0.5 wt\% of Tween 20 (polyoxyethylene sorbitan monolaurate, Sigma Chemical, St. Louis, MO), a nonionic surfactant, which prevented droplet coalescence during the experiment. Emulsion preparation consisted of mixing the three components using an Ultra-Turrax T25 high-speed blender (IKA Works Inc., Germany) at 15,000 rpm for 3 minutes and then passing the resulting dispersion through a highpressure laboratory homogenizer (Mini-Lab, type $8.30 \mathrm{H}$, Rannie, Denmark) at 400 bar. The bulk droplet size distributions were measured by light scattering (Malvern Mastersizer Model 3.01, Worcester, England), which requires dilution of the sample to avoid multiscattering effects. For the case of PGSTE measurements, manganese chloride $\left(\mathrm{MnCl}_{2}\right)$ was added to water to form a $10 \mathrm{mM}$ solution in the emulsion in order to suppress the water signal in the NMR experiment (Kroecker and Henkelman, 1986). It was found that the addition of such a small amount of $\mathrm{MnCl}_{2}$ did not affect the physical properties or the stability of the emulsion.

At $20^{\circ} \mathrm{C}$ the density of isooctane $\rho_{\text {oil }}$ is 0.69 $\mathrm{g} / \mathrm{cm}^{3}$, and the density of water $\rho_{\text {water }}$ is $1.0 \mathrm{~g} / \mathrm{cm}^{3}$. Therefore the isooctane droplets tend to rise or "cream" in a quiescent emulsion. The creaming velocity of an isolated droplet with radius a can be estimated from Stokes' equation, $\mathrm{U}_{\text {Stokes }}=(2 / 9)\left(\rho_{\text {water }}-\rho_{\text {oil }}\right) \mathrm{ga}^{2} / \mu$, where $\mathrm{a} \sim 2.5 \mu \mathrm{m}$, $\mathrm{g}=980 \mathrm{~cm} / \mathrm{s}^{2}$ and the water viscosity $\mu \sim 1 \mathrm{cP}$. Creaming velocity is on the order of $10^{-4} \mathrm{~cm} / \mathrm{s}$, three orders of magnitude smaller than the characteristic cylinder velocity $\mathrm{V}$ used in this work. The kinematic viscosity of the emulsions was measured with a Cannon-Ubblehold viscometer (Cannon Instruments Co., State College, PA) and a Gay-Lussac pycnometer (Fisher Scientific) was used to measure the density. The viscosity was obtained by multiplying the value of the kinematic viscosity by the density. The 0.3 and 0.4 oil volume fraction emulsions had viscosities of 2.5 and $3.4 \mathrm{cP}$ and average densities of 0.90 and 0.89 , respectively.

\section{MRI Experiments}

The MRI experiments were performed in a Bruker Biospec imaging system with a static magnetic field of 7 Tesla and gradient intensities of up to $95 \mathrm{G} / \mathrm{cm}$. An MRI probe was constructed in our laboratory with specialized features that enabled us to study concentrated emulsion flow. The probe was built in the form of a concentric-cylinder device with an attached NMR radio-frequency (r.f.) coil. A stepper motor generated a shear flow in the flow cell by rotating the outer cylinder with constant velocity $\mathrm{V}$ while the inner cylinder remained stationary. The inner cylinder radius $R_{i}$ is $4 \mathrm{~mm}$ and outer cylinder radius $R_{o}$ is $11 \mathrm{~mm}$ (Figure 4 ). The ratio between the axial length and the gap width is 40 . Therefore, end effects were negligible in the central region of the probe. The attached Alderman-Grant r.f. coil was designed for a resonant frequency of $300 \mathrm{MHz}$, which is the proton resonance frequency in a $7 \mathrm{~T}$ magnetic field. Further details about the probe can be found in d'Avila (2003).

Velocity profiles were measured noninvasively by time-of-flight (TOF) velocimetry. Time-of-flight images of the emulsions were obtained at an outer cylinder rotation speed of 0.1 and $0.05 \mathrm{~cm} / \mathrm{s}$ by using the pulse sequence shown in Figure 2. The repetition time ranged from 4.5 to $6.5 \mathrm{~s}$ and the echo time was $5.9 \mathrm{~ms}$. The tagged plane was $1 \mathrm{~mm}$ thick and was oriented in the vertical (sagittal) direction on the flow center line (Figure 4). The time between the saturation and the $90^{\circ}$ pulses had a value of 2.0 or $4.0 \mathrm{~s}$. The image obtained had a matrix size of $128 \times 128$, and a field-of-view of $3 \times 3 \mathrm{~cm}$. The crosssection "slice" thickness selected was $2 \mathrm{~mm}$. The total experiment time ranged from 10 to $14 \mathrm{~min}$, depending on the displacement time interval. Images were also obtained for pure water at $\mathrm{V}=0.1 \mathrm{~cm} / \mathrm{s}$.

In the PGSTE experiment, the parameter varied was the phase-encoding gradient intensity G. It was assigned 20 gradient steps ranging from 0 to 0.8 $\mathrm{T} / \mathrm{m}$. The repetition time between scans was 1.0 second, the diffusion time $\Delta$ was $400 \mathrm{~ms}$ and the gradient duration $\delta$ was $20 \mathrm{~ms}$. The direction of the diffusion gradient was in the $\mathrm{x}$ direction. The matrix size was $32 \times 32$ in the cross section, the slice thickness was $4 \mathrm{~mm}$ and the field-of-view was $3 \times 3 \mathrm{~cm}$, resulting in a voxel size of $0.94 \times 0.94 \times 4.0 \mathrm{~mm}$. The choice of these parameters was based on the amount of signal attenuation that is needed to obtain the average and standard deviation of a lognormal droplet size distribution. Data was processed with a program written with Matlab 5.3 that allowed us to determine the droplet size distribution.

Chemical shift images were obtained at outer cylinder rotation speeds of $0.05 \mathrm{~cm} / \mathrm{s}$ by using a spinecho pulse sequence with phase-encoding gradients 
indicating the position of each pixel (Fig. 2a). The repetition time was $1 \mathrm{~s}$ and the echo time was 11.1 $\mathrm{ms}$. The image obtained had a matrix size of $32 \times 32$, and a field-of-view of $3 \times 3 \mathrm{~cm}$. The cross-sectional "slice" thickness selected was $1 \mathrm{~cm}$. The total experiment duration was $17 \mathrm{~min}$. The volume fraction maps were obtained by processing the CSI data using software written with Matlab 5.3.

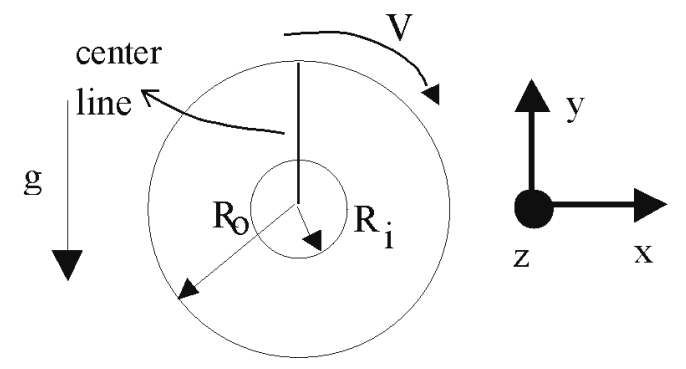

Figure 4: Cross-section of the concentric cylinders of outer cylinder radius $R_{\mathrm{o}}$ and inner cylinder radius $\mathrm{R}_{\mathrm{i}}$.

\section{RESULTS AND DISCUSSION}

\section{Velocity Profiles for Homogeneous Emulsions}

Figure 5 shows TOF images of pure water (Fig. 5a) and an emulsion of bulk oil volume fraction $\phi_{0}=0.3$ (Fig. 5b) where the outer cylinder rotates at $\mathrm{V}=0.1 \mathrm{~cm} / \mathrm{s}$. Here, the term homogeneous refers to the case when the droplets are uniformly distributed inside the flow cell (Shapley et al., 2003). By observing the tagged line, it can be seen that the concentrated emulsion has a counterrotating flow, whereas the pure water has a corotating flow. This shows the effect that the presence of droplets has on the overall flow of the system. Figure 5c shows a volume fraction map of the oil phase during the flow shown in Figure 5b, and it can be seen that the volume fraction $\phi$ along the flow cell is almost uniform; hence no droplet segregation was induced in this type of flow.

Figure 6 shows profiles of the tangential velocity $\mathrm{v}$ obtained through image processing of the TOF images shown in Figure 5. The line corresponds to the theoretical solution of the Navier-Stokes equation for a Newtonian fluid in this geometry. As should be expected, the pure water velocity profile is well described by a Newtonian flow within the experimental error bars. This result provides evidence of the accuracy of TOF in measuring velocity profiles. For the case of the emulsion, the counterrotating flow can be observed and it can be seen that this flow differs considerably from a Newtonian fluid flow. This flow behavior has been found to be due to small buoyancy effects caused by the density difference between the continuous and dispersed phases (Shapley et al., 2003).

\section{Mixing}

When an initially creamed emulsion is subjected to a shear flow, the dispersed phase will tend to mix to homogenize the droplet distribution. Figure 7 shows the volume fraction distribution obtained by CSI in the cross section of the flow cell at different times for an initially creamed emulsion of $\phi_{0}=0.3$ where the outer cylinder velocity $\mathrm{V}=0.05 \mathrm{~cm} / \mathrm{s}$. The mixing behavior of the dispersed phase can be observed, with mixing occurring due to depletion of the dispersed phase from the top creamed layer. Figure 7 also contains velocity profiles at different times during the process shown in Figure 6, showing that only the region close to the outer cylinder has flow. This mixing behavior is different from the conventional ones observed at high Reynolds numbers, where mixing proceeds through the "stretch and folding" of fluid regions (Ottino et al., 2000). In the case presented here, there is evidence that buoyancy effects have an important effect on the mixing behavior in this type of flow and the spatial distribution of droplet size might have a key role in the mixing mechanism. A more thorough analysis of this newly observed phenomenon can be found in d'Avila et al. (2003). 


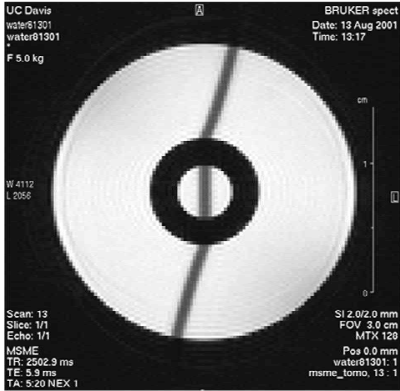

(a)

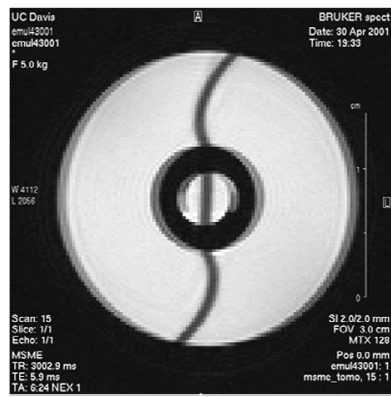

(b)

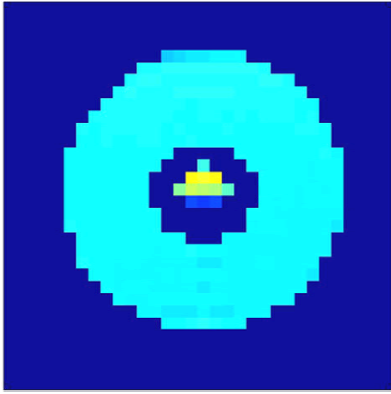

(c)

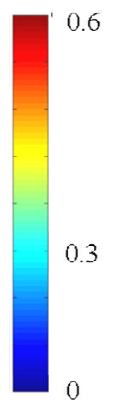

$\phi$

Figure 5: Images obtained for pure water an emulsion of bulk volume fraction $\phi=0.3$ : a) TOF image for pure water, b) TOF image for emulsion and c) CSI image.

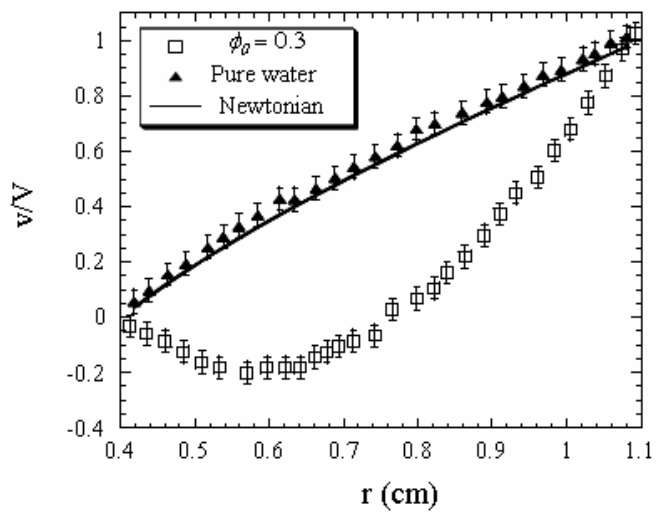

Figure 6: Velocity profiles obtained from TOF experiment: (a) pure water and (b) emulsion.

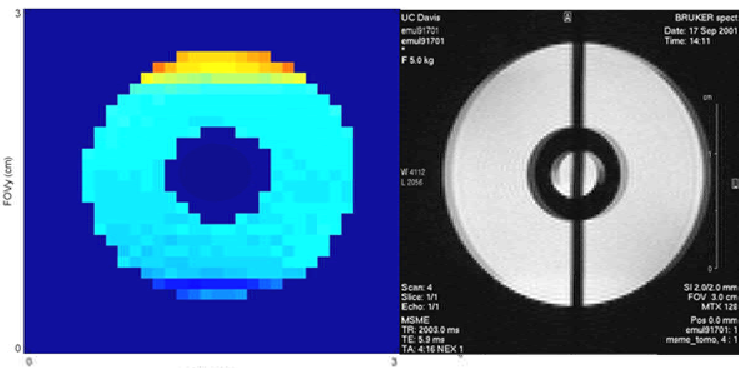

(a)

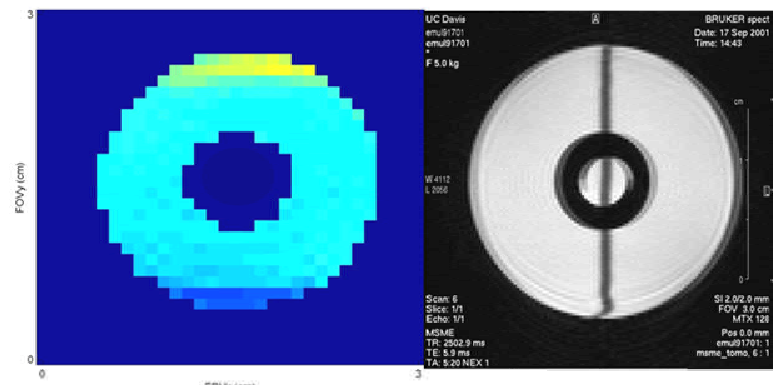

(b)

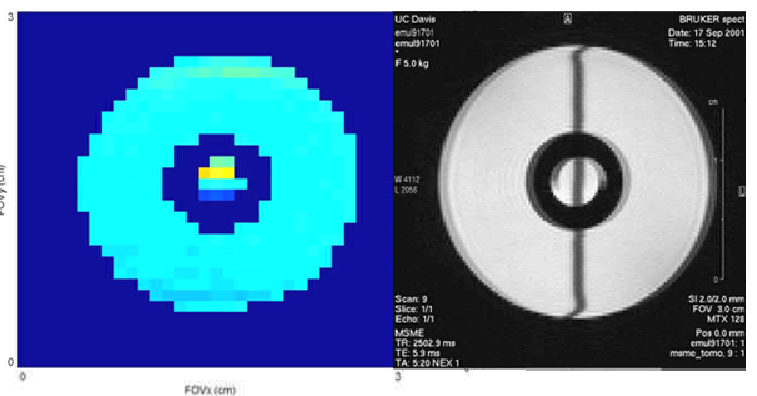

(c)

Figure 7: CSI and TOF images during mixing of an emulsion of $\phi_{0}=0.3$ and $V=0.05 \mathrm{~cm} / \mathrm{s}$. (a) $t=0$, (b) $t=9 \mathrm{~min}$ and (c) $\mathrm{t}=27 \mathrm{~min}$. The grayscale in the CSI images is the same as that shown in Figure 5. 


\section{Spatial Distribution of Droplet Sizes}

The bulk droplet size distribution was obtained for the case of a fully creamed emulsion. The signal at each pixel was used to obtain an average of all pixels inside the probe. For this case, we did not observe any signal in the phase image, which means that no flow artifacts were detected. Therefore, it was assumed that the signal was due to restricted diffusion only. Figure 8 shows the NMR data obtained and the fitting using equations (1) to (3). It can be seen that the agreement between the signal attenuation function and the experimental data is excellent. Figure $6 \mathrm{~b}$ shows a comparison between the droplet size distribution obtained by NMR and light scattering. A good agreement between the two techniques can be observed. The fact that only one peak is found in the NMR measurements is due to the a priori assumption of a lognormal distribution (section 2). However, the accuracy of static lightscattering measurements does not assure that a bimodal distribution is in fact present, also due to the limitations of this technique (Lee, 1995).

Figure 9 shows a spin echo image of the cross section of the probe for a fully creamed emulsion of $\phi_{0}=0.4$. This image was obtained by nulling the power of the saturation pulse shown in Figure 1 for a static flow cell. In this image two distinct layers can be seen; one is oil rich and the other is depleted of oil, where no NMR signal is detected.

Curves of normalized signal intensity $S / \mathrm{S}_{0}$ versus gradient intensity $\mathrm{G}$ along a horizontal line are shown in Figure 10a. The coordinates are shown in Figure 9. It can be clearly seen that the signal attenuation curves are similar, showing that along a horizontal line in a fully creamed emulsion, the droplets have essentially the same size. On the other hand, along a vertical line, it can be seen from Figure $10 \mathrm{~b}$, that the signal attenuation curves are different at different positions in the $\mathrm{y}$ direction. Therefore, a droplet size gradient is found only in the y direction for a fully creamed emulsion. Lines in Figure 10b correspond to the fit of the signal attenuation function, assuming a lognormal distribution. It can be seen that the curve fits the data very well.

From the lognormal distribution function obtained through the fitting of the signal attenuation curves, the volume average radius $\mathrm{a}_{\mathrm{avg}}$ was calculated according to $\mathrm{a}_{\text {avg }}=\int \mathrm{a}^{3} \mathrm{P}(\mathrm{a}) \mathrm{da} / \int \mathrm{a}^{2} \mathrm{P}(\mathrm{a}) \mathrm{da}$, where $\mathrm{P}(\mathrm{a})$ is the lognormal distribution function. Values of the average droplet diameter $d_{a v g}$ as a function of the position in the $\mathrm{y}$ direction are shown on Figure 11. The error bars correspond to the standard deviation of the volume weighted lognormal distribution. It can be clearly seen that the biggest droplets, which are about two times bigger than the bulk average diameter, are located at the top of the creamed layer. The average droplet diameter monotonically increases from the bottom of the creamed layer to a maximum on the top of the creamed layer. This gradient in droplet size is explained as being due to the higher rise velocity of the bigger droplets, which will tend to reach the top of the creamed layer more rapidly than the smaller ones. This experiment substantiates the capability of MRI to obtain droplet size distribution at different locations inside the sample.

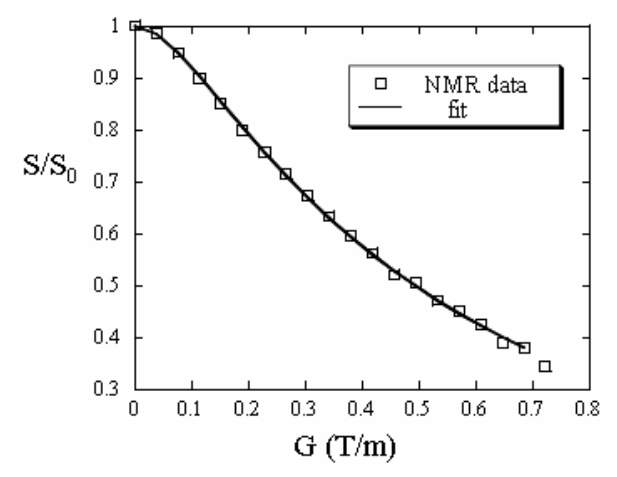

(a)

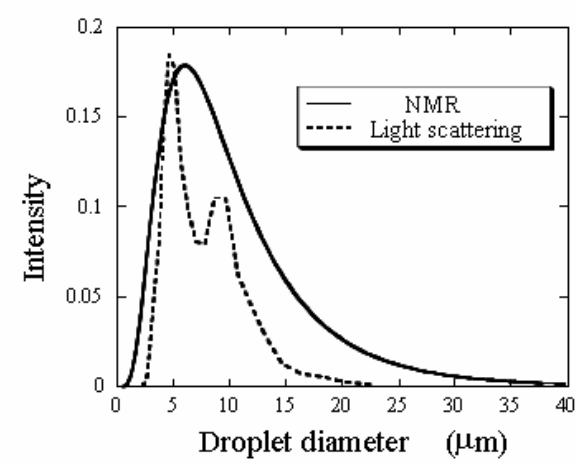

(b)

Figure 8: Bulk droplet size distribution: (a) NMR data (square) and the corresponding curve fitting (line) and (b) comparison between NMR and light scattering showing a very good agreement between the two techniques. 


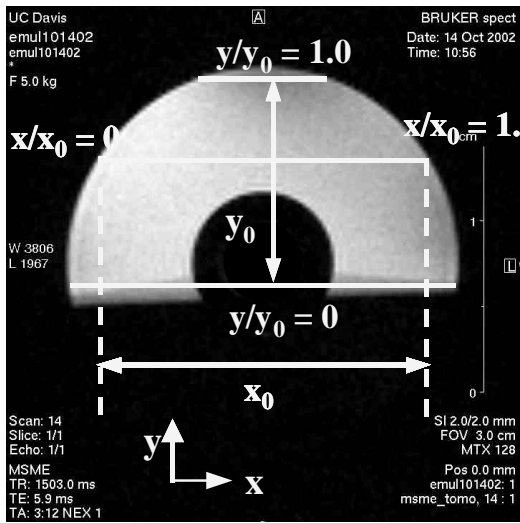

Figure 9: Spin-echo image of a fully creamed emulsion with the definition of the $\mathrm{x}$ and $\mathrm{y}$ coordinates.

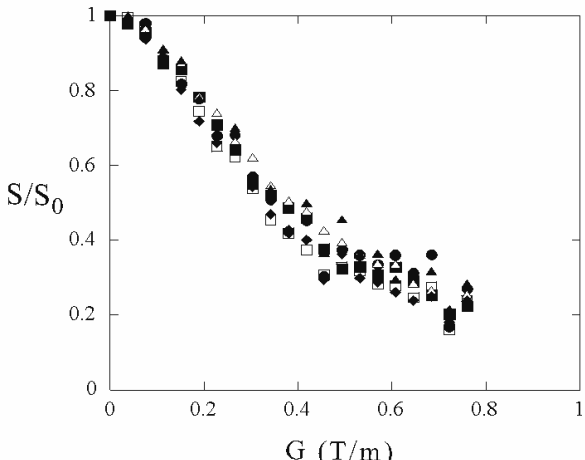

(a)

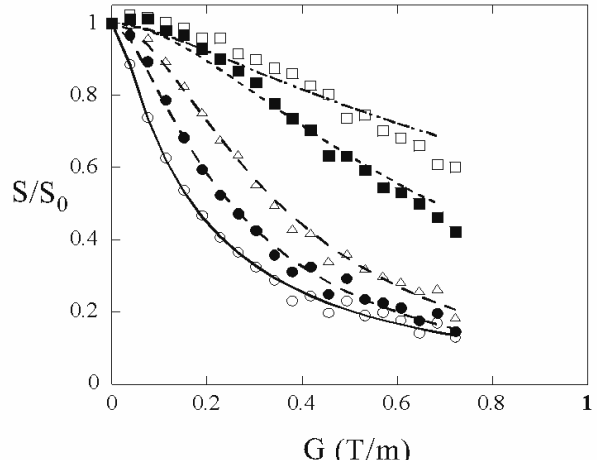

(b)

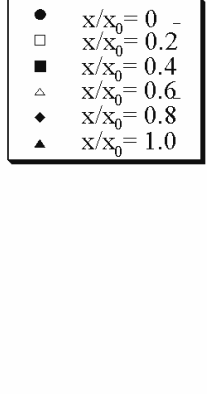

Figure 10: Signal attenuation curves at various positions along (a) a horizontal line and (b) along a vertical line inside the creamed layer. It can be seen that there is a variation in droplet size only in the y direction.

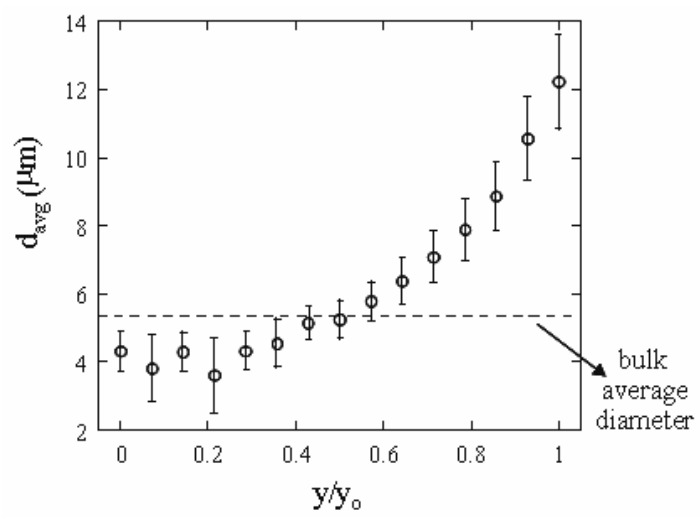

Figure 11: Plot of $d_{\text {avg }}$ versus $y / y_{0}$ for a fully creamed emulsion. It can be seen that the bigger droplets are on the top of the creamed layer.

\section{CONCLUSIONS}

In this article, the unique capabilities of MRI to study flow and microstructure of a model oil-inwater concentrated emulsion are demonstrated by presentation and discussion of experimental data using an original experimental MRI setup. Noninvasive and suited to opaque systems, MRI techniques allow measurement of velocity profiles, spatial homogeneity and spatially resolved droplet size distributions of concentrated emulsions in the same equipment. The methods presented here can be easily applied in the study of other types of multiphase systems.

Time-of-flight methods were shown to be able to measure velocity profiles for homogeneous 
concentrated emulsions. The experimental velocity profiles in oil-in-water emulsions determined in horizontal concentric cylinders revealed the occurrence of a counterrotating profile which is markedly different from the single-phase Newtonian case. It was also demonstrated that the MRI technique is appropriate for generating data in order to build volume fraction maps in emulsified systems, showing the variation of both local concentration and velocity during a mixing process. Spatially resolved droplet size distributions were also measured from MRI experiments, particularly giving the spatial dependence in the vertical y direction of droplet sizes after a creaming process.

The recent recognition of the great potential of this technique in $\mathrm{R} \& \mathrm{D}$ of products and processes and its success in handling engineering problems characterize a promising trend for the future of MRI applied to chemical engineering. We consider this article to be a contribution by presenting the unique features of MRI for the study of flow of concentrated emulsions, still a challenging problem in the field of multiphase flows.

\section{NOMENCLATURE}

a Droplet radius, $\quad \mathrm{m}$

$\bar{a} \quad$ Mean radius, $\quad \mathrm{m}$

$\mathrm{a}_{\text {avg }} \quad$ Volume average radius, $\mathrm{m}$

D Self-diffusion coefficient, $\quad \mathrm{m}^{2} / \mathrm{s}$

$\mathrm{d}_{\text {avg }} \quad$ Volume average diameter, $\mathrm{m}$

g Gravitational acceleration, $\mathrm{m} / \mathrm{s}^{2}$

G Pulsed gradient magnitude, $\quad \mathrm{T} / \mathrm{m}$

$\mathrm{G}_{\mathrm{x}}$ Magnetic field gradient in $\mathrm{x} T / \mathrm{m}$ direction,

$\mathrm{G}_{\mathrm{y}}$ Magnetic field gradient in $\mathrm{y} T / \mathrm{m}$ direction,

$\mathrm{G}_{\mathrm{z}} \quad$ Magnetic field gradient in $\mathrm{z} \quad \mathrm{T} / \mathrm{m}$ direction,

$\mathrm{P}(\mathrm{a}) \quad$ Lognormal distribution function, $\quad$ (-)

$\mathrm{R}_{\mathrm{i}} \quad$ Inner cylinder radius, $\mathrm{m}$

$\mathrm{R}_{\mathrm{o}} \quad$ Outer cylinder radius, $\mathrm{m}$

$S \quad$ Signal intensity,

$\mathrm{S}_{0} \quad$ Signal intensity at $\mathrm{G}=0$,

$\mathrm{T}$ Time, $\mathrm{s}$

V Outer cylinder velocity, $\mathrm{m} / \mathrm{s}$

\section{Greek Letters}

$\alpha_{\mathrm{m}} \quad$ Bessel function equation roots (Eq. 2), $\quad \mathrm{m}^{-1}$

$\gamma_{\mathrm{G}} \quad$ Gyromagnetic ratio, $\quad \mathrm{s}^{-1} \mathrm{~T}^{-1}$

$\delta \quad$ Gradient pulse duration,
$\Delta \quad$ Diffusion time, $\mathrm{S}$

$\phi \quad$ Local volume fraction,

$\phi_{0} \quad$ Bulk volume fraction,

$\mu \quad$ Continuous phase viscosity,

$\mathrm{Pa} \mathrm{s}$

$\rho_{\text {oil }} \quad$ Isooctane density, $\mathrm{kg} / \mathrm{m}^{3}$

$\rho_{\text {water }}$ Water density, $\mathrm{kg} / \mathrm{m}^{3}$

$\sigma \quad$ Standard deviation, $\quad \mathrm{m}$

\section{ACKNOWLEDGEMENTS}

The authors gratefully acknowledge financial support of this work received from the National Aerounautics and Space Administration NASA (NRA-96-HEDS-01), CNPq - Conselho Nacional de Desenvolvimento Científico e Tecnológico (200382/97-7) and FAPESP - Fundação de Amparo à Pesquisa do Estado de São Paulo (03/01892-0). Partial funding was provided by NSF - National Science Foundation grant CTS-98126.

\section{REFERENCES}

Abbott, J.R., Tetlow, N., Graham, A.L., Altobelli, S.A., Fukushima, E., Mondy, L.A. and Stephens, T.S., Experimental Observations of Particle Migration in Concentrated Suspensions - Couette Flow, J. Rheol. 35, no 5, 773 (1991).

Ambrosone, L., Ceglie, A., Colafemmina, G. and Palazzo, G., General Methods for Determining the Droplet Size Distribution in Emulsion Systems, J. Chem. Phys. 110, no 2, 797 (1999).

Arola, D.F., Barrall, G.A., Powell, R.L., McCarthy, K.L. and McCarthy, M.J., Use of Nuclear Magnetic Resonance Imaging as a Viscometer for Process Monitoring. Chem. Eng. Sci. 52, no 13, 2049 (1997).

Axel, L., Shimakawa, A. and MacFall, J., A Time of Flight Method of Measuring Flow Velocity by Magnetic Resonance Imaging. Mag. Res. Imag. 4, no 3, 199 (1986).

Binks, B.P., Emulsions - Recent Advances and Understanding, in Modern Aspects of Emulsion Sience, Ed. by B. P. Binks. The Royal Society of Chemistry, Cambridge (1998).

Bobroff, S. and Phillips, R.J., Nuclear Magnetic Resonance Imaging Investigation of Sedimentation of Concentrated Suspensions in Non-Newtonian Fluids, J. Rheol. 42, no 6, 1419 (1998).

Britton, M. M., Mair, R. W., Lambert, R. K. and Callaghan, P. T., Transition to Shear Banding in 
Pipe and Couette Flow of Wormlike Micellar Solutions. J. Rheol 43, no 4, 897 (1999).

Callaghan, P.T., Principles of Nuclear Magnetic Resonance Microscopy. Oxford University Press Inc., New York (1991).

Choi Y.J., McCarthy K.L., McCarthy M.J., Tomographic Techniques for Measuring Fluid Flow Properties, J. Food Sci. 67, no7, 2718(2002).

Chow, A.W., Sinton, S.W., Iwamiya, J.H. and Stephens, T.S., Shear-induced Particle Migration in Couette and Parallel-plate Viscometers: NMR Imaging and Stress Measurements. Phys. Fluids 6, no 8, 2561 (1994).

Corbett, A.M., Phillips, R.J., Kauten. R.J. and McCarthy, K.L., Magnetic Resonance Imaging of Concentration and Velocity Profiles of Pure Fluids and Solid Suspensions in Rotating Geometries. J. Rheology 39, no5, 907 (1995).

d'Avila, M.A., Flow and Characterization of Emulsions by Nuclear Magnetic Resonance. PhD dissertation, University of California at Davis (2003).

d'Avila, M.A., Shapley, N.C., Walton, J.H., Dungan, S.R., Phillips, R.J. and Powell, R.L., Mixing of Concentrated Emulsions Measured by Nuclear Magnetic Resonance Imaging. Phys. Fluids 15, no 9, 2499 (2003).

Fukushima, E., Nuclear Magnetic Resonance as a Tool to Study Flow, Ann. Rev. Fluid Mech. 31, 95 (1999).

Gladden, L.F., Magnetic Resonance: Ongoing and Future Role in Chemical Engineering Research. AIChE J. 49, no 1, 2 (2003).

Goudappel, G.J.W., van Duynhoven, J.P.M. and Mooren, M.M.W., Measurement of Oil Droplet Size Distributions in Food Oil/Water Emulsions by Time Domain Pulsed Field Gradient NMR, J. Coll. Int. Sci. 239, no 2, 535 (2001).

Hall, L.D., Amin, M.H.G., Evans, S., Nott, K.P. and Sun, L., Magnetic Resonance Imaging for Industrial Process Tomography, J. Electron. Imag. 10, no 3, 601 (2001).

Hedin, N., Furo, I, Ostwald Ripening of an Emulsion Monitored by PGSE NMR, Langmuir 17, no 16, 4746 (2001).

Hollingsworth, K.G. and Johns, M.L., Measurement of Emulsion Droplet Sizes Using PFG NMR and Regularization Methods. J. Coll. Int. Sci. 258, no 2, 383 (2003).

Jeong, E.K., Altobelli, S.A. and Fukushima, E., NMR Imaging Studies of Stratified Flows in a Horizontal Rotating Cylinder, Phys. Fluids 6, no 9, 2901 (1994).
Kroecker, R.M. and Henkelman, R.M., Analysis of Biological NMR Relaxation Data with Continuous Distributions of Relaxation Times. J. Mag. Res. 69, no 2, 218 (1986).

Lee, H.-Y., Characterization of Emulsion Stability by Nuclear Magnetic Resonance. MS thesis, University of California-Davis, 1995.

Lee, H.-Y., McCarthy, M.J. and Dungan, S.R., Experimental Characterization of Emulsion Formation and Coalescence by Nuclear Magnetic Resonance Restricted Diffusion Techniques. J. Am. Oil Chem. Soc. 75, no 4, 463 (1998).

Li, T.-Q., Seymour, J.D., McCarthy, M.J., McCarthy, K.L., Ödberg, L. and Powell, R.L., Turbulent Pipe-flow Studied by Time-averaged NMR Imaging - Measurements of Velocity Profile and Turbulent Intensity, Magn. Reson. Imag. 12, 923 (1994).

Loewenberg, M., Numerical Simulation of Concentrated Emulsion Flow, Trans. ASME 120, no 4, 824 (1998).

Mair, R.W., Hurlimann, M.D., Sen, P.N., Schwartz, L.M. and Walswothrt, R.L., Tortuosity Measurement and the Effects of Finite Pulse Widths on Xenon Gas Diffusion NMR Studies of Porous Media, Mag. Res. Imag. 19, no 3-4, 345 (2001).

Mantle, M.D. and Sederman, A.J., Dynamic MRI in Chemical Process and Reaction Engineering, Prog. Nucl. Mag. Res. Spec. 43, no 1-2, 3 (2003).

Mishra, V., Kresta, S.M. and Masliyah, J.H., Selfpreservation of the Drop Size Distribution Function and Variation in the Stability Ratio for Rapid Coalescence of a Polydisperse Emulsion in a Simple Shear Field. J. Coll. Int. Sci. 197, no 1, 57 (1998).

McCarthy, K.L., Lee, Y., Green, J. and McCarthy, M.J., Magnetic Resonance Imaging as a Sensor System for Multiphase Mixing. Appl. Mag. Res. 22, no 2, 213 (2002).

Newling, B., Gibbs, S.J., Hall, L.D., Haycock, D.E., Frith, W.J. and Ablet, S., Chemically Resolved NMR Velocimetry. Chem. Eng. Sci. 52, no 13, 2059 (1997).

Newling, B., Glover, P.M., Keddie, J.L., Lane, D.M. and McDonald, P.J., Concentration Profiles in Creaming Oil-in-Water Emulsion Layers Determined with Stray Field Magnetic Resonance Imaging, Langmuir 13, no 14, 3621 (1997).

Otsubo, Y. and Prud'homme, R.K., Rheology of Oilin-Water Emulsions. Rheol. Acta 33, no 4, 29 (1994).

Ottino, J.M., DeRoussel, P., Hansen, S. and Khakhar, D.V., Mixing and Dispersion of 
Viscous Liquids and Powdered Solids. Advances in Chemical Engineering 25, 105 (2000).

Packer, K.J., Magnetic Resonance in Porous Media: Forty Years on. Mag. Res. Imag 21 no 3-4, 163 (2003).

Packer, K.J. and Rees, C., Pulsed NMR Studies of Restricted Diffusion. 1. Droplet Size Distributions in Emulsions, J. Coll. Int. Sci. 40, no 2, 206 (1972).

Pope, J.M. and Yao, S., Quantitative NMR Imaging of Flow, Conc. Mag. Res. 5, 281 (1993).

Phillips, R.J., Armstrong, R.C., Brown, R.A., Graham, A.L. and Abbott, J.R., A Constitutive Equation for Concentrated Suspensions that Accounts for Shear-induced Particle Migration. Phys. Fluids A 4, no 1, 30 (1992).

Riess, J.G., Blood Substitutes and Other Potential Biomedical Applications of Fluorinated Colloids. J. Fluor. Chem. 114, no 2, 119 (2002).

Rumpel, H. and Pope, J.M., Chemical Shift Imaging in Nuclear Magnetic Resonance: A Comparison of Methods, Conc. Magn. Reson. 5, 43 (1993).

Salmon, J.B., Manneville, S. and Colin, A., Shear Banding in a Lyotropic Lamellar Phase. I. Timeaveraged Velocity Profiles, Phys. Rev. E 68, no 5, art 051503 (2003).

Seymour, J.D., Manz, B. and Callaghan, P.T., Pulsed Gradient Spin Echo Nuclear Magnetic Resonance Measurements of Hydrodynamic Instabilities with Coherent Structure: Taylor Vortices, Phys. Fluids 11, no 5, 1104 (1999).
Seymour, J.D., Maneval, J.E., Powell, R.L., McCarthy, K.L. and McCarthy, M.J., NMR Velocity Phase Encoded Measurements of Fibrous Suspensions, Phys. Fluids A 5, no 11, 3010 (1993).

Shapley, N.C., d'Avila, M.A., Walton, J.H., Powell, R.L., Dungan, S.R. and Phillips, R.J., Complex Flow Transitions in a Homogeneous, Concentrated Emulsion, Phys. Fluids 15, no 4, 881 (2003).

Slichter, C. P., Principles of Magnetic Resonance, $3^{\text {rd }}$ ed., Springler, New York (1990).

Tirumkudulu, M., Tripathi, A. and Acrivos, A., Particle Segregation in Monodisperse Sheared Suspensions, Phys. Fluids 11, 507 (1999).

Uludag, Y., Barrall, G.A., Arola, D.F., McCarthy, M.J. and Powell, R.L., Polymer Melt Rheology by Magnetic Resonance Imaging, Macromol. 34, no 16, 5520 (2001).

Velankar, S., Van Puyvelde, P., Mewis, J. and Moldenaers, P., Effect of Compatibilization on the Breakup of Polymeric Drops in Shear Flow, J. Rheol. 45, no 4, 1007 (2001).

Walkenstrom, P. and Hermansson, A.M., Microstructure in Relation to Flow Processing, Curr. Op. Coll. Int. Sci. 7, no 5-6, 413 (2002).

Zinchenko, A.Z. and Davis, R.H., Shear Flow of Highly Concentrated Emulsions of Deformable Drops by Numerical Simulations, J. Fluid Mech. 455, 21 (2002). 